\title{
Fully-distributed Load Frequency Control Strategy in an Islanded Microgrid Considering Plug-In Electric Vehicles
}

\author{
Xiao Qi ${ }^{1,2, *(1)}$, Yan Bai ${ }^{1}$, Huanhuan Luo ${ }^{3}$, Yiqing Zhang ${ }^{2}$, Guiping Zhou ${ }^{3}$ and Zhonghua Wei ${ }^{3}$ \\ 1 School of Control and Computer Engineering, North China Electric Power University, Beijing 102206, China; \\ by@ncepu.edu.cn \\ 2 Synergy Innovation Center for Energy Economics of Shandong, Shandong Institute of Business and \\ Technology, Yantai 264005, China; zyq_751105@163.com \\ 3 State Grid Liaoning Electric Power Supply Co., Ltd., Shenyang 110004, China; rawn@163.com (H.L.); \\ 18900911559@163.com (G.Z.); wzh_fw@ln.sgcc.com.cn (Z.W.) \\ * Correspondence: qixiao@ncepu.edu.cn; Tel.: +86-010-6177-2102
}

Received: 22 May 2018; Accepted: 19 June 2018; Published: 20 June 2018

\begin{abstract}
With large-scale integration of electric vehicles, this paper investigates the load frequency control problem in an islanded microgrid with plug-in electric vehicles (PEVs), which can be regarded as mobile battery energy storages to provide a valuable contribution to frequency regulation. A novel fully-distributed control strategy is proposed to achieve fast frequency regulation of islanded microgrids and effective coordination control of distributed energy sources. Firstly, distributed control based on an improved linear active disturbance rejection algorithm is realized through a multi-agent system, and it greatly enhances the anti-disturbance capability of the microgrid. Then, in order to guarantee the effectiveness of PEVs in frequency regulation, PEVs are controlled following the controllable power rate (CPR) calculated from the consensus-based multi-agent system. Furthermore, the system control construction in this paper is well designed to avoid the negative effects caused by system communication time delay. Finally, numerical simulations under different disturbances are carried out to demonstrate the effectiveness of the proposed control strategy in comparison with other previous control strategies.
\end{abstract}

Keywords: islanded microgrid; load frequency control; fully-distributed control; linear active disturbance rejection algorithm; plug-in electric vehicles

\section{Introduction}

With the increasing penetration of renewable energy sources (RESs), the microgrid, which is regarded as a promising approach to integrate various distributed generation resources with the power grid, is attracting more interest from scholars. However, the extensive use of electronic power converters poses new challenges to microgrid operation and control due to the significant decrease of system rotating inertia. The occurrence of power deficiency or shortage will result in large frequency fluctuation. Especially when the microgrid operates in islanded mode, system frequency stability is under great threat due to the intermittency and stochasticity of RESs and load demands. Therefore, in order to maintain the stability of system frequency, numerous energy storages are integrated with the microgrid, which also increase system cost and scale. Nevertheless, the plug-in electric vehicles can be regarded as mobile battery energy storages and provide a valuable contribution to frequency regulation. Considering the more complicated topological structure of the microgrid, it is of significant importance to design more effective control strategies to ensure the system frequency stability. 
Traditionally, the centralized control strategy (CCS) has been widely employed to deal with the load frequency control (LFC) problem [1-3]. However, the single-point failure of the central controller may break down the entire system. Furthermore, the implementation of CCS strongly depends on the computational capability of the central controller and the communication capability of the network. The CCS requires global information of each component of the microgrid. That is to say, in addition to the time delay caused by the calculation process, the communication delay is negligible. The significant time delay directly brings great challenges to the islanded microgrid in realizing rapid frequency regulation. In the past few years, many scholars have devoted themselves to improving system frequency stability by reducing negative the influences caused by communication and calculation time delays $[4,5]$.

Different from CCS, the distributed control strategy (DCS) only requires information collected through a local communication network. It is more flexible and reliable in satisfying the requirements of the microgrid, especially a microgrid with a high penetration of renewable energy sources. Therefore, due to its excellent characteristics including high reliability and flexibility, low cost and high computational efficiency, DCS is more suitable to solve the LFC problem in the microgrid. Furthermore, the multi-agent system (MAS) is widely employed in the distributed control strategy [6-10], and it is also adopted in this paper. Researchers have proposed various distributed control strategies based on MAS to maintain the frequency stability of the microgrid with plug-in electric vehicles. In [11], a novel PEV charging model for frequency regulation was proposed to maximize PEVs' total utility and to satisfy individual PEVs' daily drive patterns simultaneously. A fully-distributed control scheme is proposed to economically share wind power fluctuations among multiple PEVs and realize flexible frequency regulation. In [12], a distributed peer-to-peer multi-agent framework is proposed to maintain the supply-demand balance in the microgrid, and the PEVs are considered as destributed energy storages (DESs). In [13], the proposed cooperative distributed control strategy for PEVs maximizes the welfare and satisfaction of PEV customers while considering the charging constraints. The frequency stability is well ensured in the aforementioned literature; however, it can be further improved by designing more efficient control strategies.

In fact, although the aggregates of PEVs can be regarded as a special kind of battery energy storage system, the output power of PEVs is stochastic and fluctuating. That is to say, it is almost impossible for PEVs to be controlled the same as the battery energy storage system to provide accurate performance. Therefore, a novel fully-distributed load frequency control strategy for the islanded microgrid with the contribution of plug-in electric vehicles is proposed in this paper. First of all, this paper designs local controllers for PEVs to eliminate frequency deviation. A new performance indicator named the controllable power rate (CPR) is proposed to coordinate the PEVs' output power, and the CPR is calculated through consensus-based MAS. The advantages of this design in comparison to other works is as follows: The CPR is only used to dispatch the total power that needs to be adjusted, that is to say the communication time delay of CPR does not affect the control activity of local controllers. It can be obviously found that the negative effect of time delay is well avoided, and the frequency stability is greatly enhanced. This is also the major contribution of this paper. To the authors' best knowledge, few studies have been carried out with the consideration of controlling the stochastic and fluctuating power of PEVs. Except the proposed CPR, this paper also employs an improved linear active disturbance rejection control (ILADRC) algorithm [14] because of its excellent robustness and anti-disturbance capacity. The stochastic and fluctuating power of PEVs can be regarded as system external disturbance, then it can be estimated by the linear extended state observer and compensated by the well-designed control law. Finally, a two-layer parallel MAS framework consisting of a plug-in electric vehicle multi-agent system (PEV-MAS) and a diesel generator multi-agent system (DG-MAS) is designed to realize distributed control of the islanded microgrid. Under this system, the PEVs can operate at the maximum charging rate most of the time. The output power of PEVs only contributes to the frequency regulation process, and then, the changed power of PEVs will be compensated by the 
DGs. However, most of the previous works only considered the contribution of PEVs to frequency regulation and neglected people's requirements of quick charging.

Numerical simulations are carried out to demonstrate the effectiveness of the proposed control strategy. The controller parameters are optimized by genetic algorithm-based particle swarm optimization (GA-based PSO). Simulations are based on the MATLAB/Simulink (R2017b, The MathWorks, Inc., Natick, MA, USA) environment.

The rest of this paper is organized as follows. The fundamental theories of the consensus algorithm and ILADRC algorithm are described in Section 2. The dynamic models of the islanded microgrid are introduced in Section 3. Then, the proposed control strategy is described in Section 4. Section 5 presents the simulation results and analysis. Finally, conclusions are drawn in Section 6.

\section{Fundamental Theory}

This section introduces the fundamental theory. The first part briefly introduces the theory of a consensus-based multi-agent system. The second part describes the theory of ILADRC.

\subsection{Theory of a Multi-Agent System}

A network is usually expressed as $\mathcal{G}=(\mathcal{N}, \mathcal{E})$, where $\mathcal{N}=1,2, \cdots, n$ represents the set of nodes, $\mathcal{E}$ represents the set of edges and edge $(i, j) \in \mathcal{E}$ represents the connected nodes. As for node $i$, its initial value is $x_{i}(0) \in R$, and $x(0)=\left(x_{1}(0), x_{2}(0), \cdots, x_{n}(0)\right)^{T}$ represents the vector of the initial values on the whole network. The MAS aims to compute the average value $\frac{1}{n} \sum_{i=1}^{n}\left(x_{i}(0)\right)$ following different dynamics. Based on the consensus algorithm, the average value $\frac{1}{n} \sum_{i=1}^{n}\left(x_{i}(0)\right)$ can be obtained by communicating with neighbor nodes. The distributed linear iterations are described by the following equation.

$$
x_{i}(t+\tau)=w_{i i} x_{i}(t)+\sum_{j \in \mathcal{N}_{i}} w_{i j} x_{j}(t)
$$

where $t=0,1,2, \cdots$ and $w_{i j}$ is the communication weight on $x_{j}$ at node $i$.

This iteration can be expressed as the following form.

$$
x(t+\tau)=W x(t)
$$

where:

$$
W=\left(\begin{array}{cccc}
w_{11} & w_{12} & \cdots & w_{1 n} \\
w_{21} & w_{22} & \cdots & w_{2 n} \\
\vdots & \vdots & \ddots & \vdots \\
w_{n 1} & w_{n 2} & \cdots & w_{n n}
\end{array}\right)
$$

The value of $W$ determines the converging speed of MAS. Therefore, for the purposes of providing convergence guarantee and an appropriate converging speed, the improved metropolis algorithm [13] is employed in this paper. The $w_{i j}$ is given by the following equation.

$$
w_{i j}= \begin{cases}\frac{2}{n_{i}+n_{j}+1}, & j \in N_{i} \\ 1-\sum_{j \in \mathcal{N}_{i}} \frac{2}{n_{i}+n_{j}+1}, & i=j \\ 0, & \text { otherwise. }\end{cases}
$$

where $\mathcal{N}_{i}$ represents the neighbor agents of agent $i$ and $n_{i}$ and $n_{j}$ represent the number of neighbors of agents $i$ and $j$, respectively.

\subsection{Theory of Improved Linear Active Disturbance Rejection Algorithm}

The complexity of the microgrid makes it necessary to employ more effective controllers. With the outstanding robustness and powerful anti-disturbance ability, the linear active disturbance rejection 
control algorithm (LADRC) is widely adopted. In order to enhance the frequency stability, this paper adopts an improved linear active disturbance rejection algorithm [14], which has been studied in the authors' previous work. The ILADRC algorithm has a simple construction, a fast response speed and strong anti-interference capability.

Supposethat the controlled process is as:

$$
\ddot{y}=b u+f(\dot{y}, y, u, d)
$$

where $y$ is the output signal of system, $u$ is the output signal of controller, $d$ is the external disturbance, $b$ is the process parameter with the estimation value of $b_{0}$ and $f$ is the total disturbance with the combination of external and internal disturbance.

The state space equation is as Equation (10). The linear extended state observer (LESO) is designed to estimate the value of $y, \dot{y}$ and $f$, which is described as Equation (11).

$$
\begin{gathered}
\left\{\begin{array}{l}
\dot{x_{1}}=x_{2} \\
\dot{x_{2}}=x_{3}+b_{0} u \\
\dot{x_{3}}=\dot{f} \\
y=x_{1}
\end{array}\right. \\
\left\{\begin{array}{l}
\dot{z}_{1}=z_{2}+\beta_{1}\left(y-z_{1}\right) \\
\dot{z_{2}}=z_{3}+\beta_{2}\left(y-z_{1}\right)+b_{0} u \\
\dot{z_{3}}=\beta_{3}\left(y-z_{1}\right)
\end{array}\right.
\end{gathered}
$$

where $\left[\beta_{1}, \beta_{2}, \beta_{3}\right]=\left[3 \omega_{0}, 3 \omega_{0}^{2}, \omega_{0}^{3}\right]$ and $\omega_{0}$ represents the observer bandwidth.

Then, the disturbance compensation is as Equation (12), and the control system is transformed into an integral cascade as Equation (13).

$$
\begin{gathered}
u=\frac{u_{0}-z_{3}}{b_{0}} \\
\ddot{y}=f+u_{0}-z_{3} \approx u_{0}
\end{gathered}
$$

Furthermore, the control law of ILADRC is redesigned as Equation (14), which reduces the stress of the linear extended state observer and increases the estimation accuracy by eliminating the negative effect of the estimation error of $z_{1}$ and $z_{2}$.

$$
u_{0}=k_{p}(r-y)-k_{d} \dot{y}
$$

\section{Simulation Model}

This paper considers the islanded microgrid with the combination of the diesel generator (DG), the variable-speed wind turbine generator (WTG), the plug-in electric vehicle (PEV) and load demand, where WTG and DG generate power for load consumption and the charging of the PEV. The configuration of the microgrid system is described in Figure 1. Due to the rapid response of PEVs' power output, the PEVs can be properly controlled to realize fast frequency recovery [15]. Then, the total power change of PEVs is compensated by DGs. Meanwhile, the variable-speed WTG participates in frequency regulation by releasing the inertia and sharing the resultant energy with the grid following the grid frequency fluctuation. 


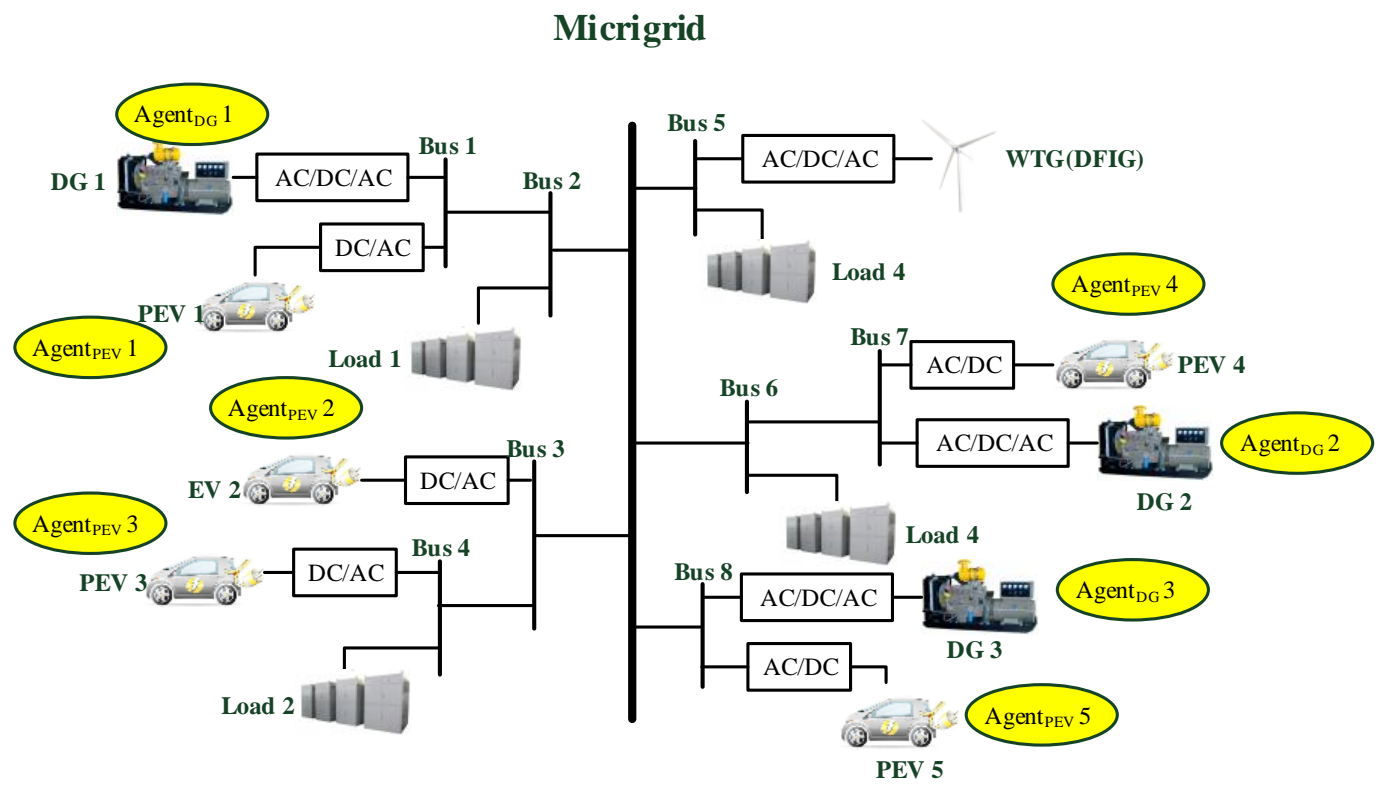

Figure 1. Microgrid architecture.

In the stable system state, the output power of each component satisfies the following equation:

$$
P_{D G}+P_{W T G}+P_{P E V}=P_{L}
$$

where $P_{W T G}$ is the output power of WTG, $P_{D G}$ is the output power of DG, $P_{P E V}$ is the output power of PEV and $P_{L}$ is the load demand.

The balance of load demand and generated power determines the frequency stability. The frequency deviation can be calculated by Equation (2) when the system power is out of balance. The deviation of the system power is calculated by Equation (3). Furthermore, the delay of the frequency characteristics is considered for the actual practice. Hence, according to [16], the Equation (2) is transformed into Equation (4):

$$
\begin{gathered}
\Delta f=\frac{\Delta P}{k} \\
\Delta P=P_{D G}+P_{W T G}+P_{P E V}-P_{L} \\
G_{s y s}=\frac{\Delta P}{\Delta f}=\frac{1}{k(T s+1)}=\frac{1}{M s+D}
\end{gathered}
$$

where $\Delta f$ and $\Delta P$ denote the frequency deviation of the microgrid and the power deviation of the microgrid, respectively. $k$ denotes the microgrid frequency characteristic constant; $G_{s y s}$ denotes the system transfer function; $M$ and $D$ are the equivalent inertia constant and damping constant of the microgrid, respectively. The values of the parameters are shown in Table 1 [17].

Table 1. System and diesel generator (DG) model parameters used for simulation.

\begin{tabular}{ccc}
\hline Symbol & Description & Value \\
\hline$M$ & Power system inertia constant & 0.1667 \\
$D$ & Power system damping constant & 0.015 \\
$T_{G}$ & Valve devicetime constant & $0.1 \mathrm{~s}$ \\
$T_{T}$ & Diesel generator time constant & $8 \mathrm{~s}$ \\
\hline
\end{tabular}




\subsection{Diesel Generator Model}

The diesel generator is an important component of the islanded microgrid power system, as it provides the main output power and compensates the system power deviation to ensure the stability of the microgrid power system. In this paper, a second-order lag transfer function model of DG is used for simulation, which can almost describe the actual model [17]. The transfer function is described by Equation (5), and parameter values are shown in Table 1.

$$
G_{D G}=\frac{1}{T_{G} s+1} \cdot \frac{1}{T_{T} s+1}
$$

\subsection{Variable-Speed Wind Turbine Generator Model}

The rotation speed of the wind turbine generator is decoupled from the microgrid system frequency because of the extensive application of power electronic converters, which results in the mitigation of microgrid inertia [18]. The microgrid system stability is greatly reduced due to the aforementioned situation. In order to make the WTG contribute to the frequency regulation, many effective control strategies based on variable-speed WTG have been proposed by researchers [19], one of which is described in Figure 2. The values of the parameters are shown in Table 2.

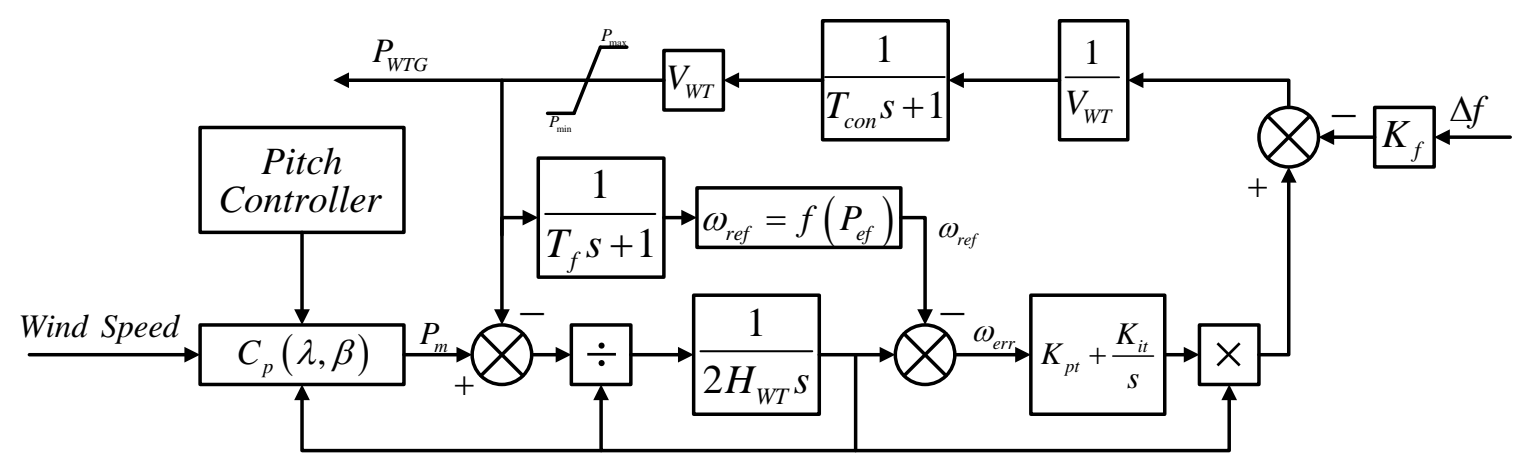

Figure 2. Variable-speed wind turbine generator model.

Table 2. Wind turbine generator (WTG) model parameters used for simulation.

\begin{tabular}{ccc}
\hline Symbol & Description & Value \\
\hline$H_{W T}$ & Wind turbine generator inertia & $5.19 \mathrm{~s}$ \\
$T_{c o n}$ & Convertor response time constant & $0.02 \mathrm{~s}$ \\
$T_{f}$ & Power measurement time constant & $5 \mathrm{~s}$ \\
$V_{W T}$ & Wind turbine generator voltage & $1.0 \mathrm{p} . u$. \\
$k_{p t}$ & Speed regulator proportional constant & 3 \\
$k_{i t}$ & Speed regulator integral constant & 0.6 \\
$k_{f}$ & Contribution coefficient & 4 \\
$P_{\max }$ & Upper limit of wind turbine generator output & 1.2 p.u. \\
$P_{\min }$ & Lower limit of wind turbine generator output & 0.1 p.u. \\
\hline
\end{tabular}

\subsection{Plug-In Electric Vehicle Model}

An equivalent PEV model is employed in this paper. Based on the charging and discharging characteristics, the equivalent PEV model can be divided into battery and charger [20]. The model used for frequency regulation [21] is described in Figure 3, where $T_{e}=1 \mathrm{~s}$ is the time constant of PEV and $u_{C}$ is the control signal from the PEV-agent. $u_{C}$ is limited between $u_{e}=0.025$ p.u. and $-u_{e}=-0.025$ p.u. $E$ is the current charging or discharging power of the PEV. $E_{\max }$ and $E_{\min }$ are the maximum and minimum controllable energy of the PEV, respectively. $\pm \delta_{e}= \pm 0.01$ p.u. are the limits of the power ramp rate. $\Delta P_{E}$ is the final output power of the PEV. 


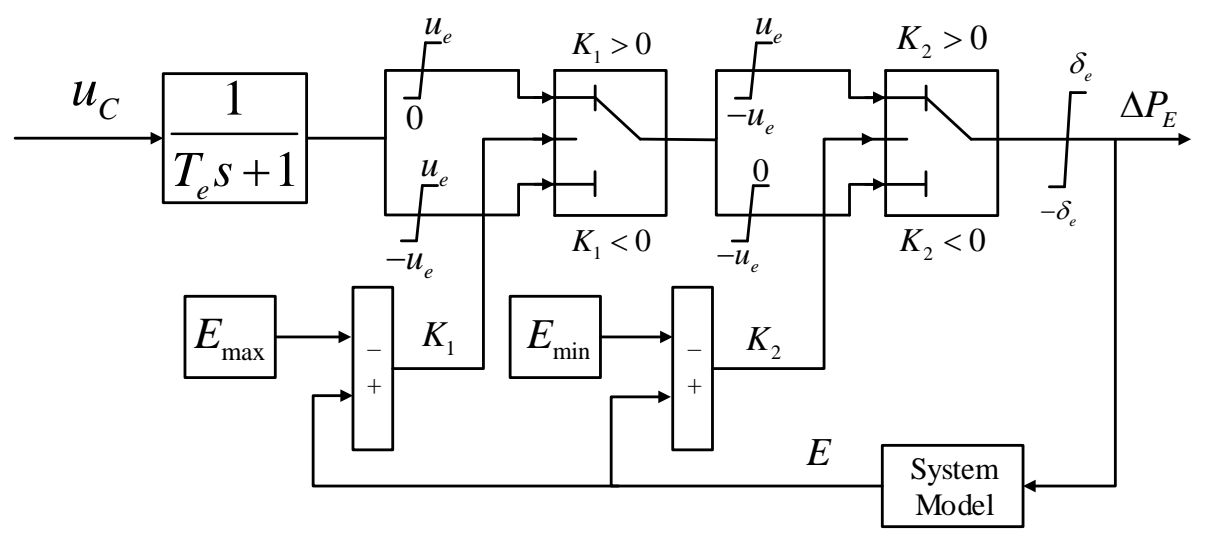

Figure 3. Plug-in electric vehicle model.

\section{Proposed Distributed Control Strategy}

Based on the aforementioned model in Section 3, the proposed distributed control strategy is introduced in detail in this section.

For the traditional microgrid, the energy storage system (ESS) mainly consists of battery energy storage, flywheel energy storage, and so on, which is usually placed centrally. Then, the power deficiency of the microgrid can be quickly compensated by ESS through the central controller. However, it is inappropriate for PEVs to be controlled by the central controller due to the decentralization of PEVs. The realization of communication among PEVs based on the central controller has a high cost. Moreover, the calculation and communication delay of the centralized control strategy adversely affects the rapid response characteristic of frequency regulation. Therefore, a novel distributed control strategy is proposed in this paper to ensure the frequency stability. This paper proposes a new performance indicator named the controllable power rate (CPR), and PEVs are controlled following the CPR calculated through consensus-based MAS. The CPR and local controllers of PEVs together protect the entire system from the negative effect caused by time delay, which is also the major advantage in comparison of other previous works. The system control construction in this paper is illustrated in Figure 4.

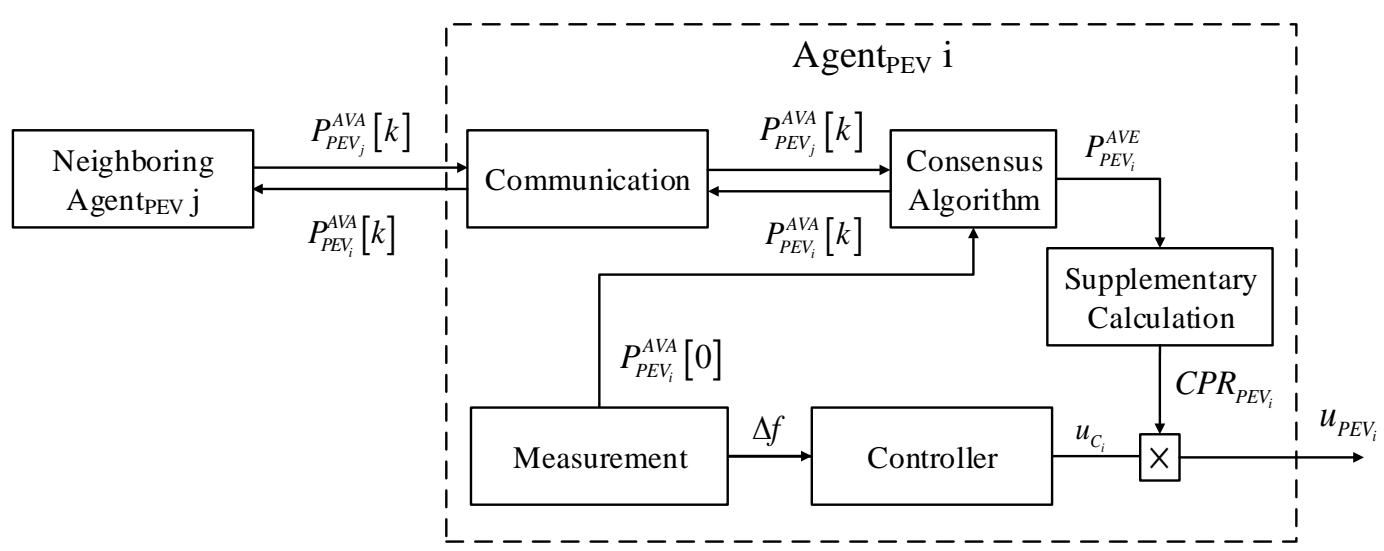

Figure 4. Agent and control structure of the plug-in electric vehicle (PEV).

The PEVs are divided into five different units according to their distance, and each unit is managed by one PEV-agent. The frequency deviation can be measured locally, and it is eliminated by the ILADRC-based controller. However, there exists the universal phenomenon that one of the PEVs cannot provide enough power to eliminate the frequency derivation based on the average algorithm. Therefore, in this paper, the controllable power rate of the PEV is calculated through the consensus 
algorithm, which is described by Equations (15) and (16), and the control signal of each PEV unit follows Equation (17). The communication topology of PEVs is described in Figure 5.

$$
\begin{gathered}
P_{P E V}^{A V E}=\frac{\sum_{i=1}^{n} P_{P E V_{i}}^{A V A}}{n} \\
C P R_{P E V_{i}}=\frac{P_{P E V_{i}}^{A V A}}{n \cdot P_{P E V_{i}}^{A V E}} \\
u_{P E V_{i}}=u_{C_{i}} \cdot C P R_{P E V_{i}}
\end{gathered}
$$

where $P_{P E V_{i}}^{A V E}$ denotes the average available power change of PEV, $P_{P E V_{i}}^{A V A}$ denotes the available power change of $P E V_{i}, u_{C_{i}}$ denotes the control signal of ILADRC-based controller and $u_{P E V_{i}}$ denotes the final control signal of $P E V_{i}$.

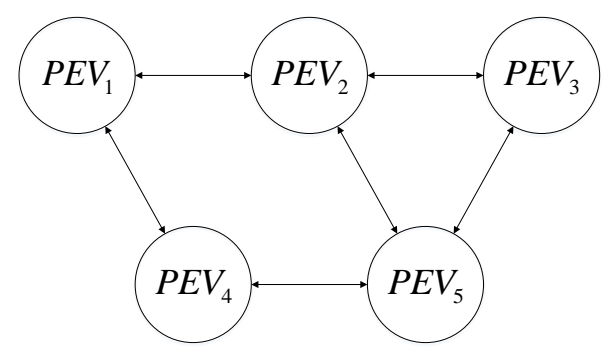

Figure 5. Communication topology of PEVs.

Based on the aforementioned control strategy, the distributed control of PEVs is realized without the negative effects caused by calculation and communication delay.

The agent and control structures of DG are illustrated in Figure 6. The total variable power of PEVs is regarded as the total reference power variation of DG, which is described as Equation (18). Based on the consensus algorithm, the reference power variation of each DG follows Equation (19), and the communication topology of DG is described in Figure 7. Subsequently, the changed power of PEVs due to the frequency regulation is completely compensated by DGs. Thus, the PEVs can operate at the maximum charging rate most of the time, and the output power of PEVs only contributes to the frequency regulation process. However, most of the previous works only consider the contribution of PEVs in frequency regulation.

$$
\begin{gathered}
\left\{\begin{array}{l}
\Delta P_{D G_{1}}^{R E F}=\Delta P_{P E V}^{1}+\Delta P_{P E V}^{2}+\Delta P_{P E V}^{3} \\
\Delta P_{D G_{2}}^{R E F}=\Delta P_{P E V}^{4} \\
\Delta P_{D G_{3}}^{R E F}=\Delta P_{P E V}^{5}
\end{array}\right. \\
\Delta P_{D G}^{R E F}=\Delta P_{D G}^{A V E}=\frac{\sum_{i}^{n} \Delta P_{D G_{i}}^{R E F}}{n}
\end{gathered}
$$

where $\triangle P_{D G_{i}}^{R E F}$ denotes the reference power variation of $D G_{i}, \triangle P_{P E V}^{i}$ denotes the variable power of the unit of $P E V_{i}$ and $P_{D G}^{A V E}$ and $\triangle P_{D G}^{R E F}$ denote the average power change of the DG and the reference power change of each DG, respectively.

Combined with the model described in Section 3 and the aforementioned control theory, the structure of the proposed microgrid frequency control strategy is shown in Figure 8. Then, the fully-distributed control of microgrid frequency regulation can be realized by this control strategy. Theoretically, the proposed control strategy can guarantee the stability of the microgrid, and its performances are better than most of the previous studies. Numerical simulations will be carried out to demonstrate the validity of the proposed control strategy in Section 5. 


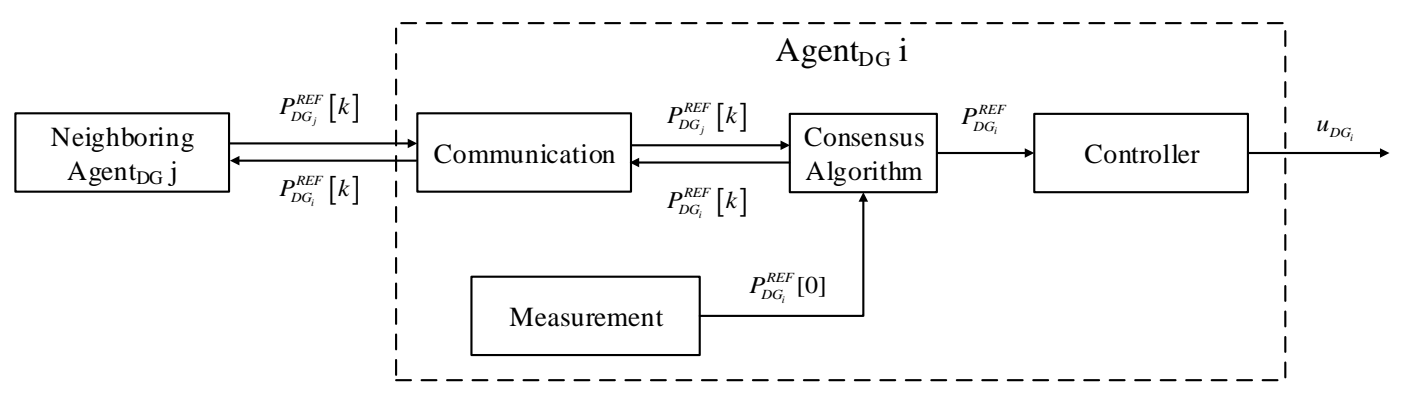

Figure 6. Agent and control structure of DG.

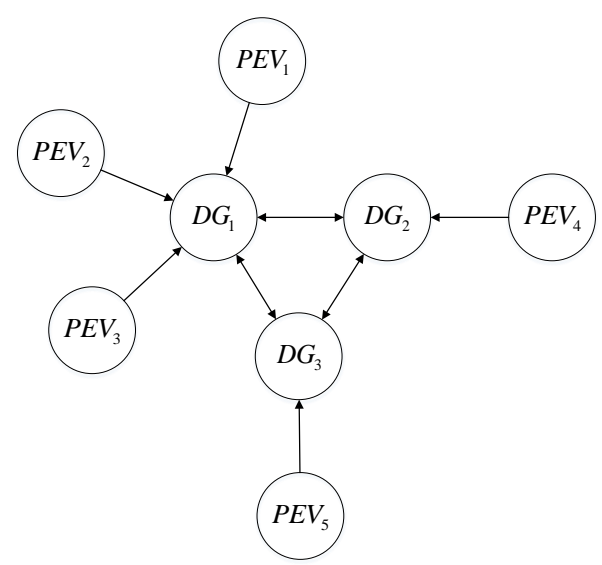

Figure 7. Communication topology of DGs.

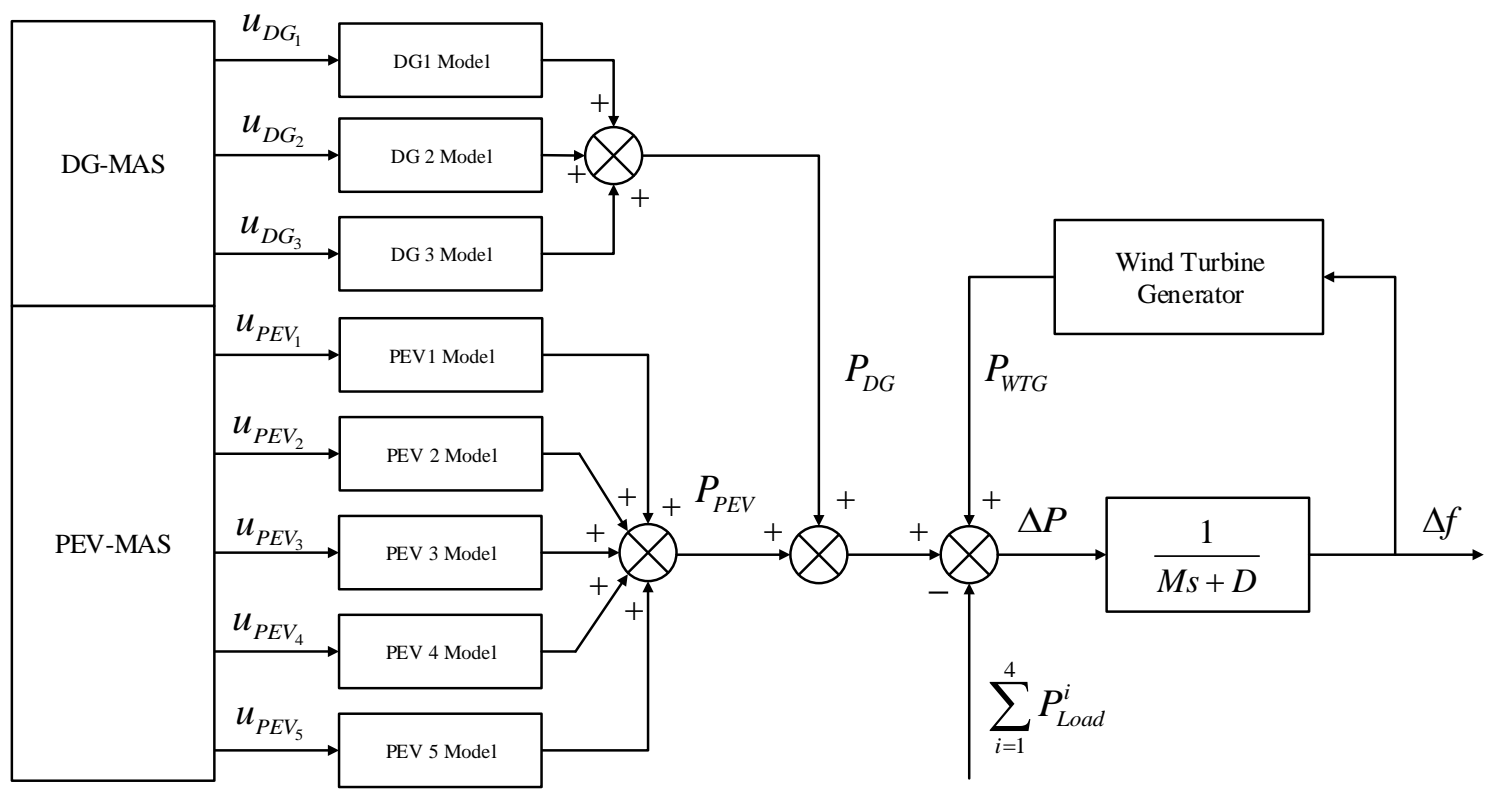

Figure 8. Control strategy of microgrid frequency regulation. Multi-agent system (MAS), multi-agent system.

\section{Simulation Results and Analysis}

At the beginning of the simulation experiments, each part of microgrid power system is operating in the steady state. The simulations are based on the MATLAB/Simulink environment, and the step time is set to $0.001 \mathrm{~s}$. Furthermore, the controller parameters are optimized by the genetic algorithm-based particle swarm optimization algorithm. The fitness function is calculated by the sum 
of the rate of change of frequency $(\mathrm{RoCoF})$ and the integral of the time-weighted absolute value of the error (ITAE), which is described by Equations (20)-(22).

$$
\begin{gathered}
\text { ITAE }=\int t|\Delta f| d t \\
\text { RoCoF }=\int t\left|\frac{d f}{d t}\right| d t \\
\text { Fitness Value }=\int t|\Delta f| d t+\int t\left|\frac{d f}{d t}\right| d t
\end{gathered}
$$

In the following part, the effectiveness of the proposed control strategy is demonstrated by two different simulation cases including: Case A: simulations are under load demand change and PEV power change without considering stochastic disturbances; Case B: simulations consider stochastic disturbances of load demand, WTG and PEV.

The parameters of CCS-ILADRC used for the simulation are shown in Table 3 and the parameters of DCS-ILADRC used for simulation are shown in Table 4.

Table 3. Parameters of the centralized control strategy (CCS)-improved linear active disturbance rejection control (ILADRC) used for the simulation.

\begin{tabular}{cccccc}
\hline \multicolumn{3}{c}{ Diesel Generator } & \multicolumn{3}{c}{ Plug-In Electric Vehicle } \\
\hline$b_{0}$ & $\omega_{0}$ & $\omega_{c}$ & $b_{0}$ & $\omega_{0}$ & $\omega_{c}$ \\
5.1482 & 17.3446 & 23.8629 & 1.8105 & 18.0748 & 7.1637 \\
\hline
\end{tabular}

Table 4. Parameters of the distributed control strategy (DCS)-ILADRC used for the simulation.

\begin{tabular}{cccccc}
\hline \multicolumn{3}{c}{ Diesel Generator } & \multicolumn{3}{c}{ Plug-In Electric Vehicle } \\
\hline$b_{0}$ & $\omega_{0}$ & $\omega_{c}$ & $b_{0}$ & $\omega_{0}$ & $\omega_{c}$ \\
8.3141 & 0.0052 & 76.8572 & 16.6795 & 0.1971 & 15.9749 \\
\hline
\end{tabular}

\subsection{Case A}

This case investigates the frequency stability under load demand change and PEV output change. The output of load demand changes from 1 p.u. to 1.1 p.u. at $1 \mathrm{~s}$, and $P E V_{1}$ output changes from 0 p.u. to 0.1 p.u. at 5 s. The initial states of DG, WTG and PEV are supposed to be stable.

Firstly, system performances based on CCS-ILADRC without time delay, CCS-ILADRC with a 10-ms delay, DCS-ILADRC without time delay and DCS-ILADRC with a 10-ms delay are studied to demonstrate the superiority of the proposed DCS-ILADRC. The transient responses of the system frequency deviation are shown in Figure 9, and the transient responses of supply-demand mismatch are shown in Figure 10. After the increase of load demand, the frequency deviation can be well eliminated. It is obvious that the system performances based on the proposed DCS-ILADRC are the best among those based on the other three control strategies.

Without considering the time delay, the settling time based on the proposed DCS-ILADRC is $0.542 \mathrm{~s}$, which is shorter than $1.490 \mathrm{~s}$ of the system based on CCS-ILADRC. The frequency overshoot and fitness value based on DCS-ILADRC are decreased to $-3.711 \times 10^{-3} \mathrm{~Hz}$ and $2.017 \times 10^{-4} \mathrm{~Hz}$, respectively. The detailed comparison results are described in Table 5. Similarly, the simulation results under the change of PEV power can also indicate the superiority of the system based on the proposed DCS-ILADRC. 


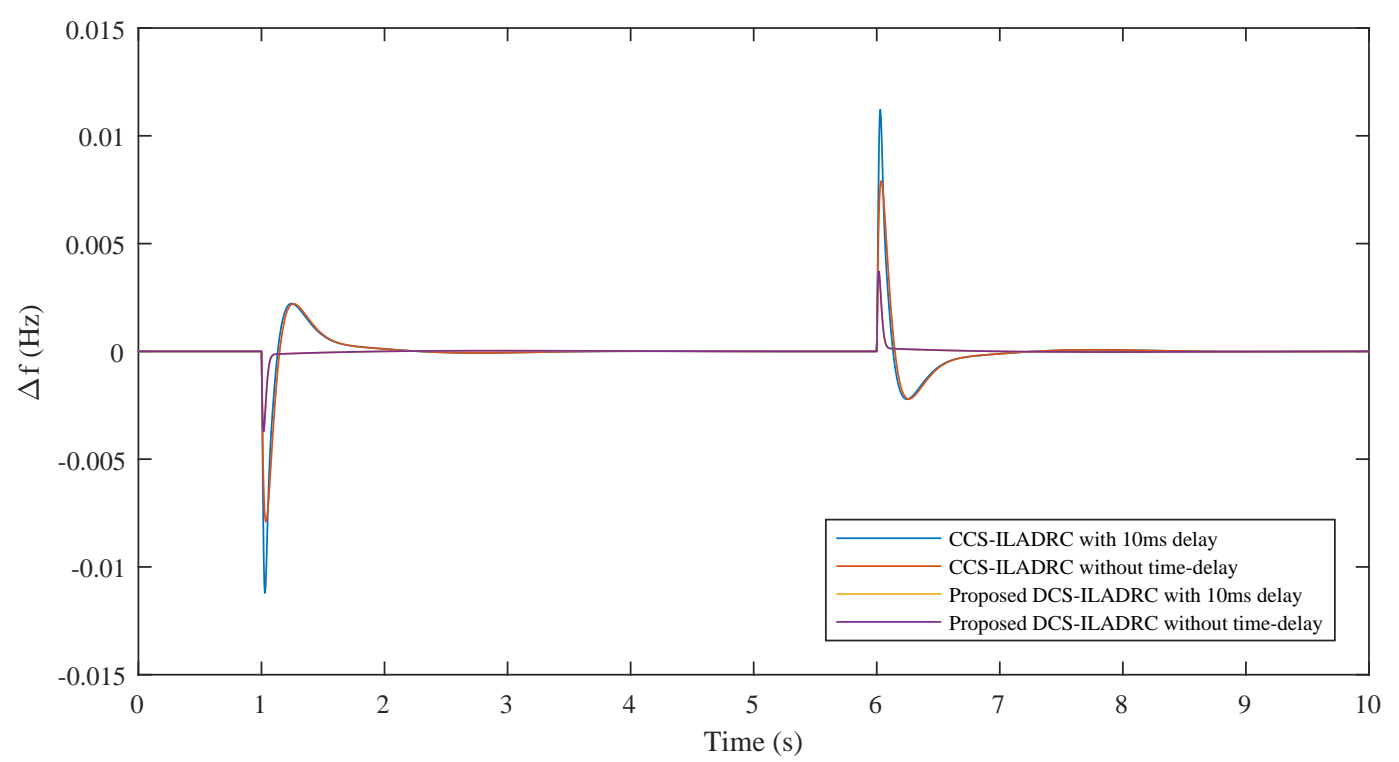

Figure 9. Transient response of the system frequency deviation.

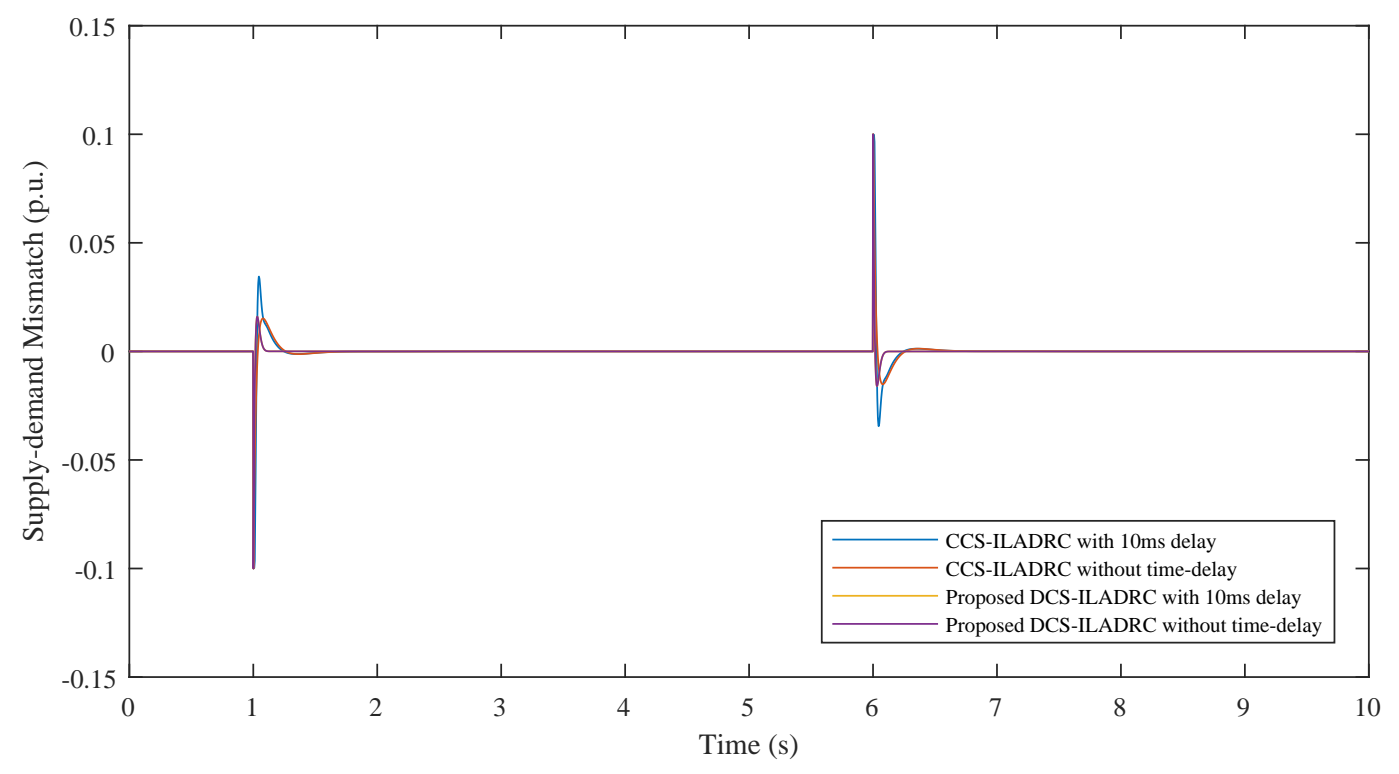

Figure 10. Transient response of supply-demand mismatch.

Table 5. Performance comparison with CCS-ILADRC without time delay, CCS-ILADRC with 10-ms delay, DCS-ILADRC without time delay and DCS-ILADRC with 10-ms delay.

\begin{tabular}{ccccc}
\hline Disturbance & Control Strategy & Overshoot & Settling Time & ITAE + RoCoF \\
\hline \multirow{5}{*}{$\Delta P_{L}=0.1$ p.u. } & CCS-ILADRC without time delay & $-7.906 \times 10^{-3} \mathrm{~Hz}$ & $1.490 \mathrm{~s}$ & $2.413 \times 10^{-2}$ \\
& CCS-ILADRC with 10-ms delay & $-1.121 \times 10^{-2} \mathrm{~Hz}$ & $1.495 \mathrm{~s}$ & $2.452 \times 10^{-2}$ \\
& DCS-ILADRC without time delay & $-3.711 \times 10^{-3} \mathrm{~Hz}$ & $0.542 \mathrm{~s}$ & $2.017 \times 10^{-4}$ \\
& DCS-ILADRC with 10-ms delay & $-3.711 \times 10^{-3} \mathrm{~Hz}$ & $0.549 \mathrm{~s}$ & $2.097 \times 10^{-4}$ \\
\hline \multirow{5}{*}{$\Delta P_{P E V_{1}}=0.1$ p.u. } & CCS-ILADRC without time delay & $7.905 \times 10^{-3} \mathrm{~Hz}$ & $1.485 \mathrm{~s}$ & $2.408 \times 10^{-2}$ \\
& CCS-ILADRC with 10-ms delay & $1.122 \times 10^{-2} \mathrm{~Hz}$ & $1.450 \mathrm{~s}$ & $2.456 \times 10^{-2}$ \\
& DCS-ILADRC without time delay & $3.712 \times 10^{-3} \mathrm{~Hz}$ & $0.544 \mathrm{~s}$ & $2.019 \times 10^{-4}$ \\
& DCS-ILADRC with 10-ms delay & $3.711 \times 10^{-3} \mathrm{~Hz}$ & $0.547 \mathrm{~s}$ & $2.086 \times 10^{-4}$ \\
\hline
\end{tabular}


Furthermore, suppose that the communication and calculation delay is $10 \mathrm{~ms}$; it is obvious that the negative effect caused by time delay can be well avoided based on the proposed DCS-ILADRC. The performances based on DCS-ILADRC with different time delays are almost unchanged. However, system performances based on CCS-ILADRC greatly depend on the communication and calculation delay. Thus, the proposed control strategy can protect the system from the time delay effect. Most of the previous works decrease the time delay effect through modifying advanced control theory or accelerating the convergent speed of MAS. However, there still exits a non-negligible negative effect caused by time delay. The proposed distributed control strategy provides a new idea for researchers.

Based on the above analysis, the frequency stability can be greatly guaranteed by the proposed control strategy. For the purpose of deeply analyzing the performances of DCS-ILADRC, the transient power outputs of load demand, WTG, DG and PEV are shown in Figures 11 and 12. Obviously, the multi-agent system of PEVs based on ILADRC can realize a fast frequency recovery. When system frequency is in a stable state, the output power of PEVs returns to its initial state by the compensation of DGs. This ensures that the PEVs can operate at the maximum charging rate most of the time. In contrast, most of the previous works only considered the contribution of PEVs to the frequency regulation, which may result in the PEVs not being able to be fully charged when people need them. Moreover, the WTG also contributes to the frequency regulation once the frequency deviation has occurred. Finally, the controllable power rate of PEVs is shown in Figure 13.
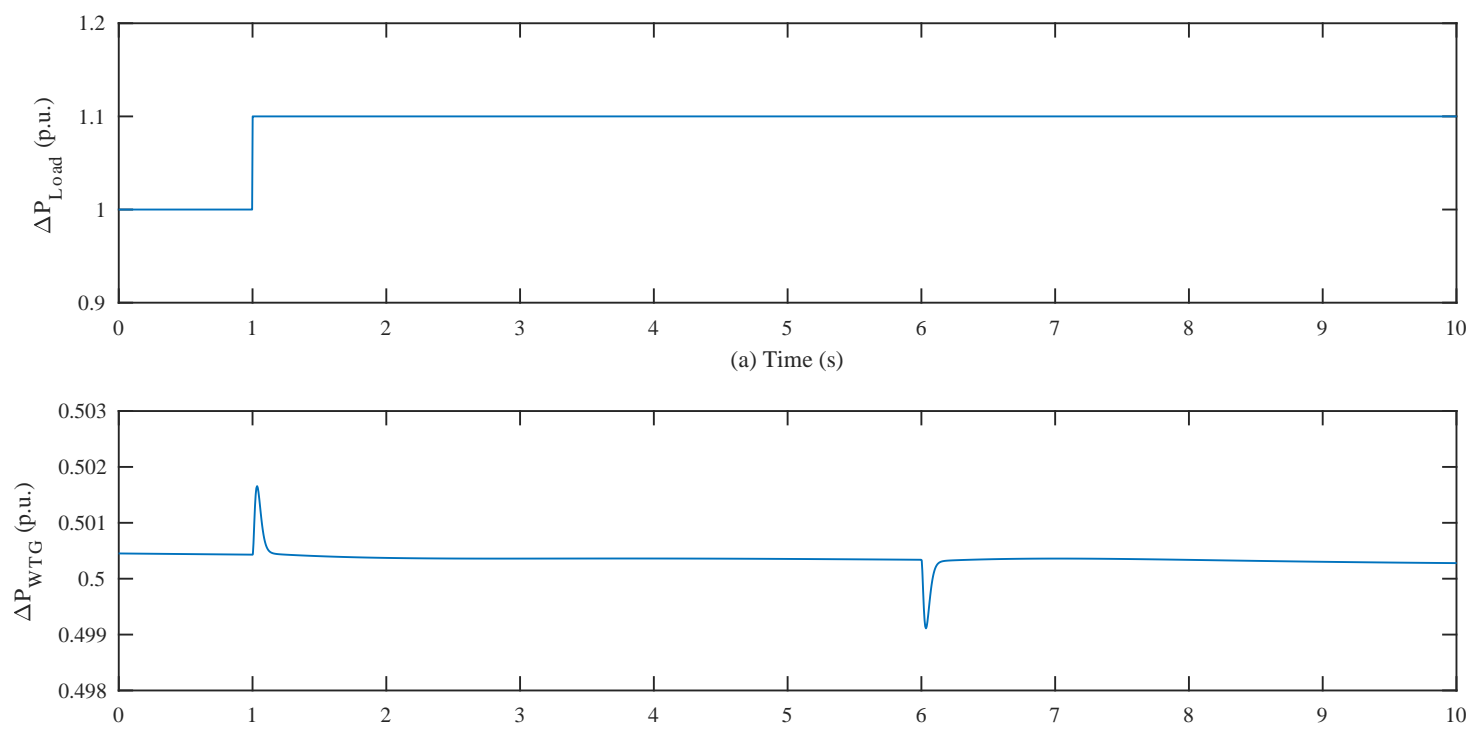

(b) Time (s)

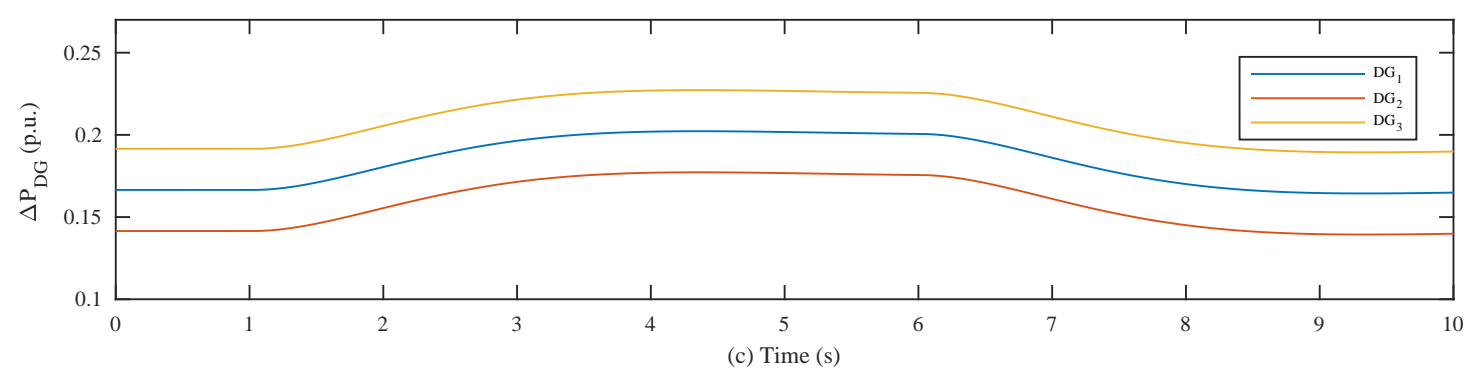

Figure 11. Transient power output of each source. (a) Load demand output; (b) power of WTG; (c) power of DG. 

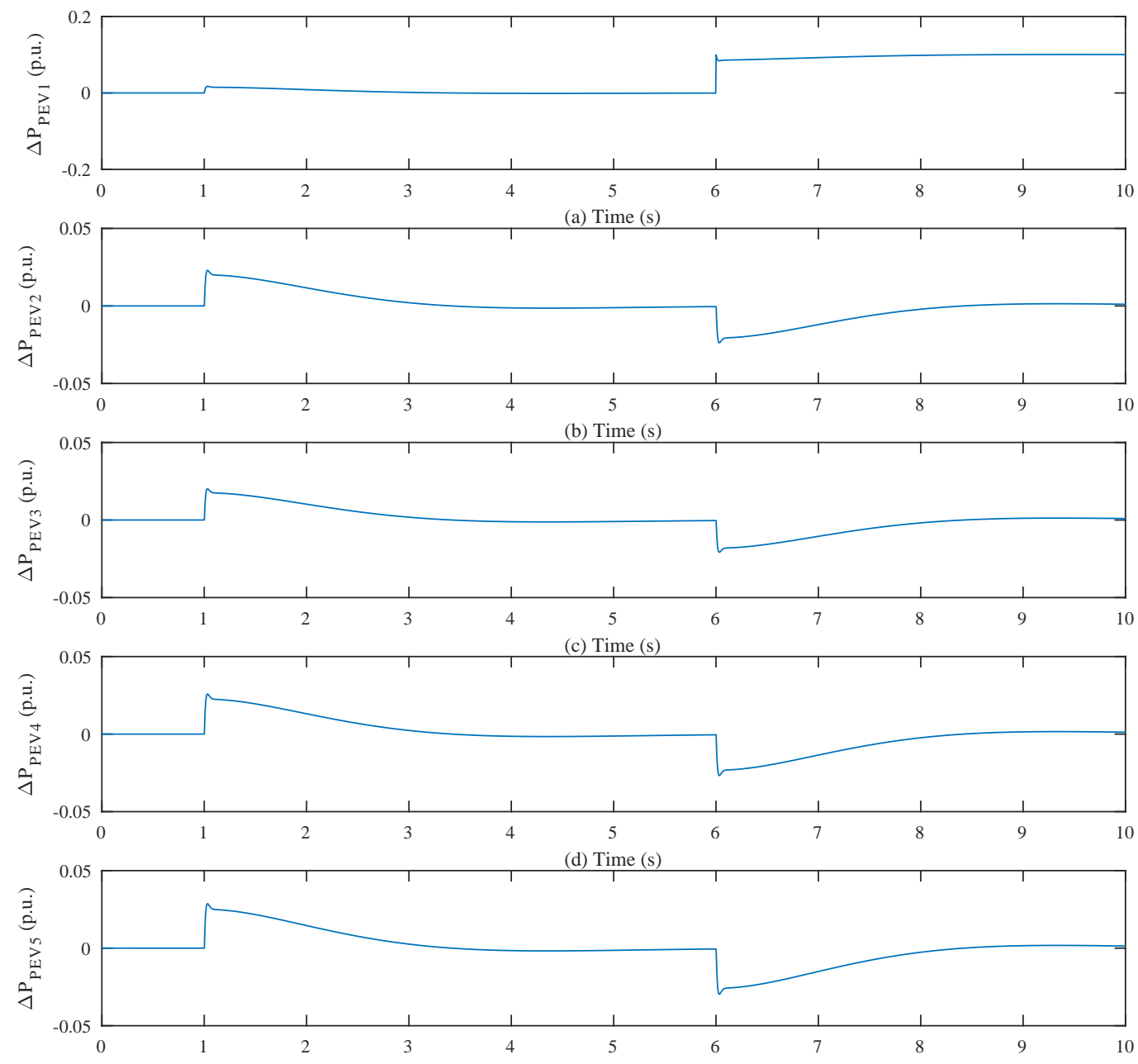

(e) Time (s)

Figure 12. Transient power output of PEV. (a) Dynamic response of $P E V_{1}$; (b) dynamic response of $P E V_{2} ;(\mathbf{c})$ dynamic response of $P E V_{3} ;$ (d) dynamic response of $P E V_{4} ;(\mathbf{e})$ dynamic response of $P E V_{5}$.

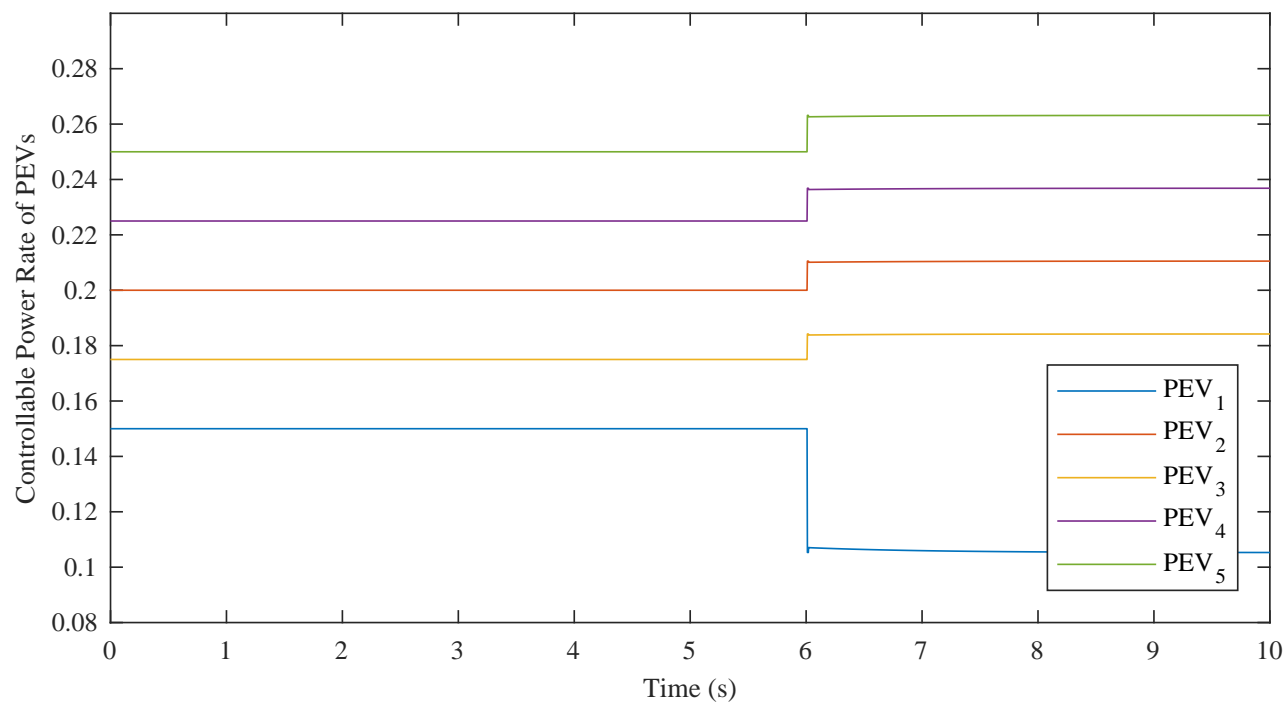

Figure 13. Controllable power rate of PEVs. 


\subsection{Case $B$}

It is of significantly practical importance to investigate the system anti-disturbance ability under the stochastic disturbance of load demand, WTG and PEVs' output, especially with the stochastic and fluctuating power of PEVs. Accordingly, this case study's frequency deviation profile is caused by stochastic changes of each system component output. The frequency transient responses and supply-demand mismatch based on different control strategies are described in Figures 14 and 15, respectively. As seen in Figure 14, the variation range of $\Delta f$ with DCS-ILADRC without time delay is between $-3.072 \times 10^{-3} \mathrm{~Hz}$ to $2.879 \times 10^{-3} \mathrm{~Hz}$, which is extremely smaller than that with CCS-ILADRC. $I T A E+R o C o F$ is reduced to 0.8546 . The comparison data are shown in Table 6 . The transient responses of the power output of each component are shown in Figures 16 and 17.

Table 6. Performance comparison considering the stochastic disturbance.

\begin{tabular}{ccc}
\hline Control Strategy & ITAE + RoCoF & Range of $\boldsymbol{\Delta f}$ \\
\hline CCS-ILADRC without time delay & 3.4533 & $-1.665 \times 10^{-1} \mathrm{~Hz}-1.288 \times 10^{-1} \mathrm{~Hz}$ \\
CCS-ILADRC with 10-ms delay & 3.7064 & $-1.741 \times 10^{-1} \mathrm{~Hz}-1.346 \times 10^{-1} \mathrm{~Hz}$ \\
DCS-ILADRC without time delay & 0.8546 & $-3.072 \times 10^{-3} \mathrm{~Hz}-2.879 \times 10^{-3} \mathrm{~Hz}$ \\
DCS-ILADRC with 10-ms delay & 0.8550 & $-3.076 \times 10^{-3} \mathrm{~Hz}-2.880 \times 10^{-3} \mathrm{~Hz}$ \\
\hline
\end{tabular}

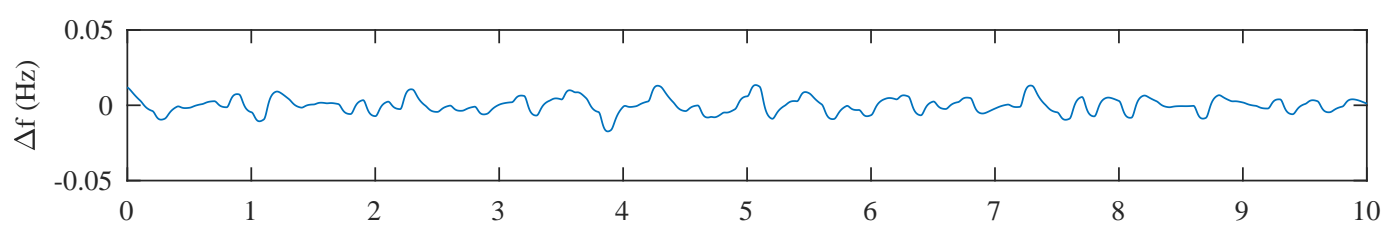

(a) Time (s)

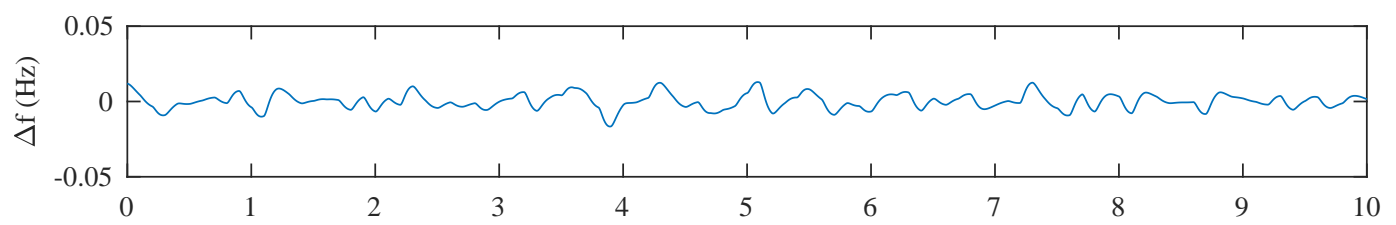

(b) Time (s)

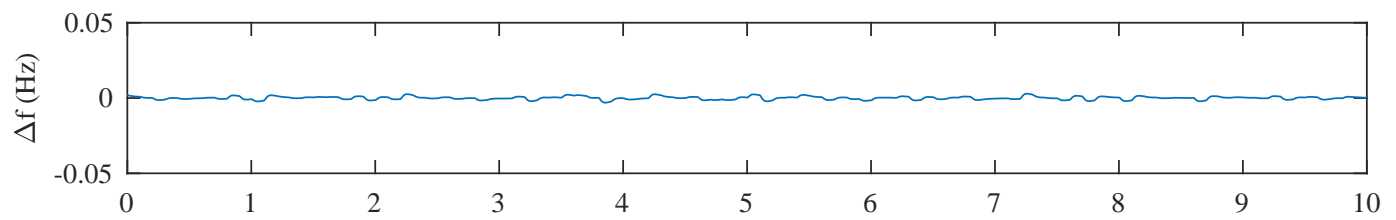

(c) Time (s)

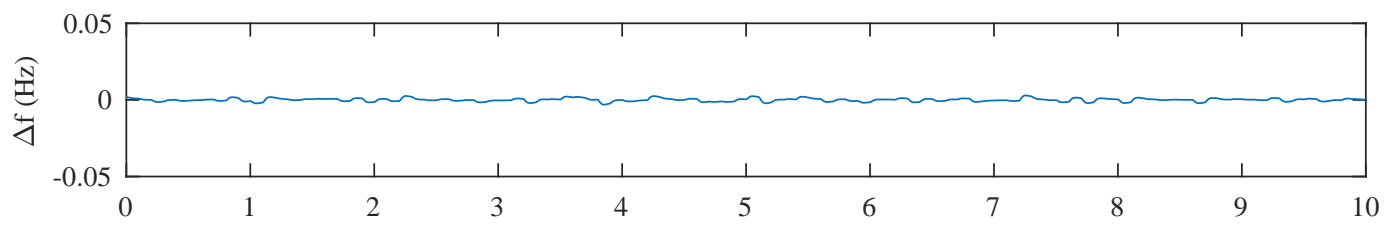

(d) Time (s)

Figure 14. Transient response of system frequency deviation with stochastic disturbance. (a) Frequency deviation based on CCS-ILADRC without time delay; (b) frequency deviation based on CCS-ILADRC with 10-ms delay; (c) frequency deviation based on DCS-ILADRC without time delay; (d) frequency deviation based on DCS-ILADRC with 10-ms delay. 


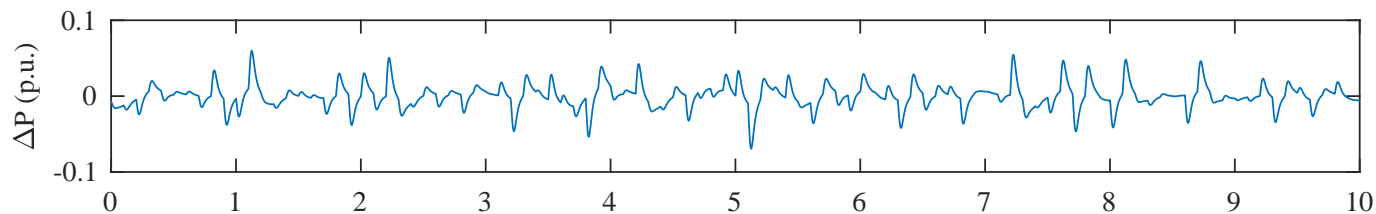

(a) Time (s)

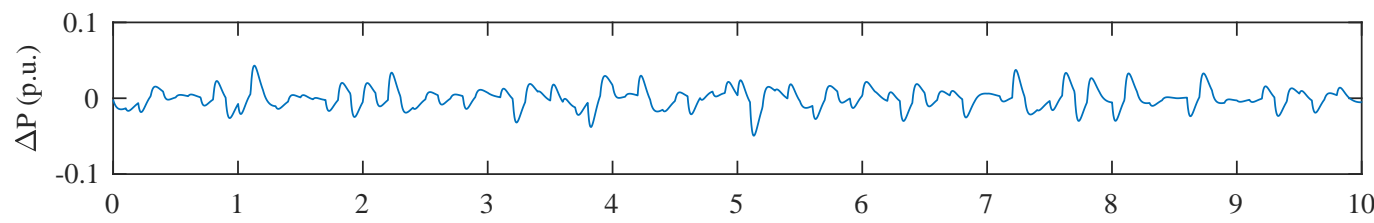

(b) Time (s)

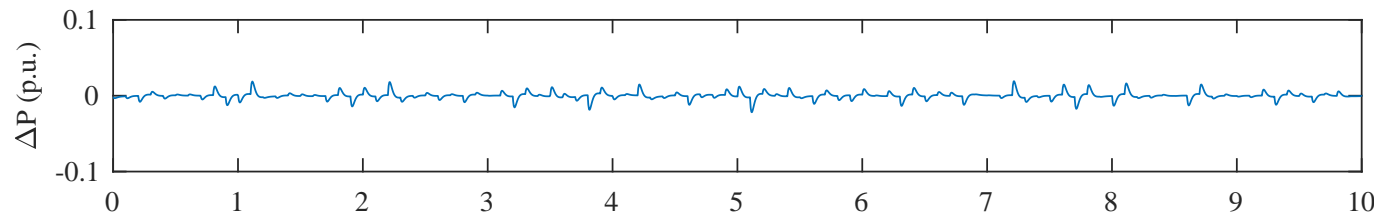

(c) Time (s)

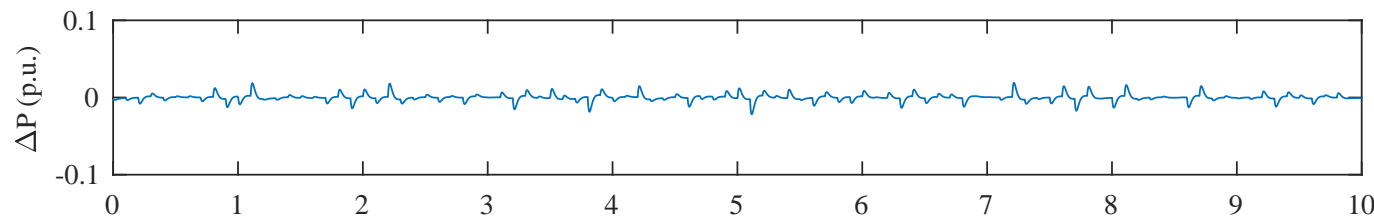

(d) Time (s)

Figure 15. Transient response of supply-demand mismatch with stochastic disturbance. (a) Supplydemand mismatch based on CCS-ILADRC without time delay; (b) supply-demand mismatch based on CCS-ILADRC with 10-ms delay; (c) supply-demand mismatch based on DCS-ILADRC without time delay; (d) supply-demand mismatch based on DCS-ILADRC with 10-ms delay.

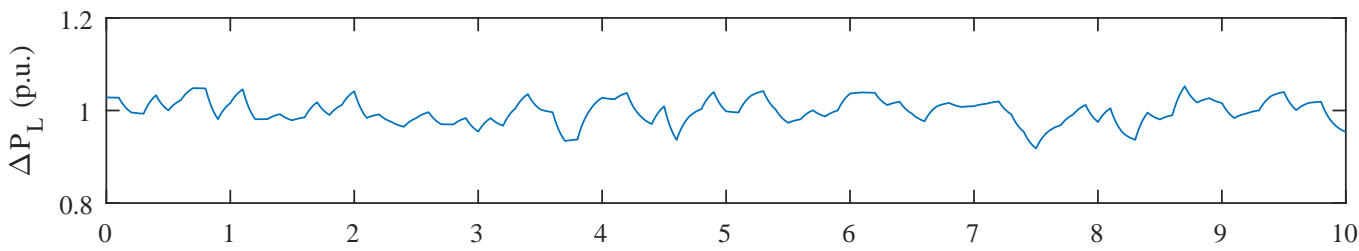

(a) Time (s)

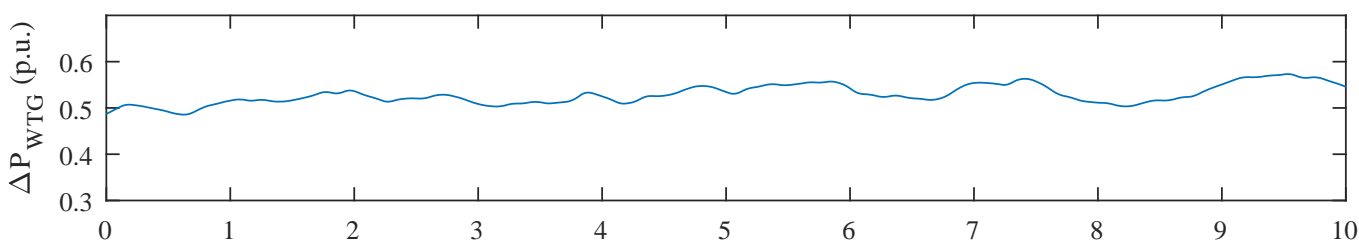

(b) Time (s)

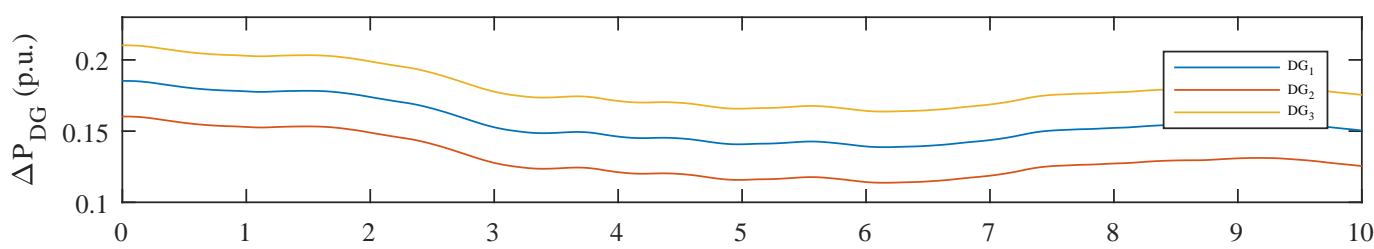

(c) Time (s)

Figure 16. Transient power output of each source with stochastic disturbance. (a) Load demand output; (b) dynamic response of WTG; (c) dynamic response of DG. 


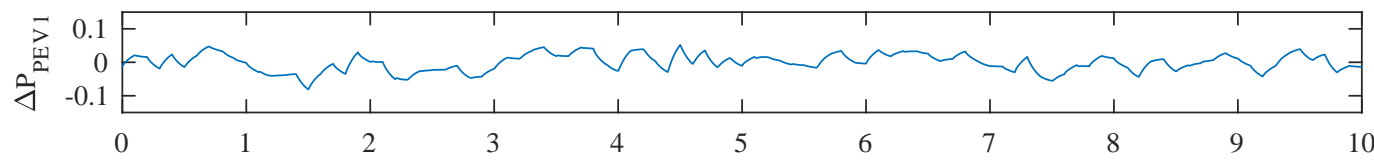

(a) Time (s)

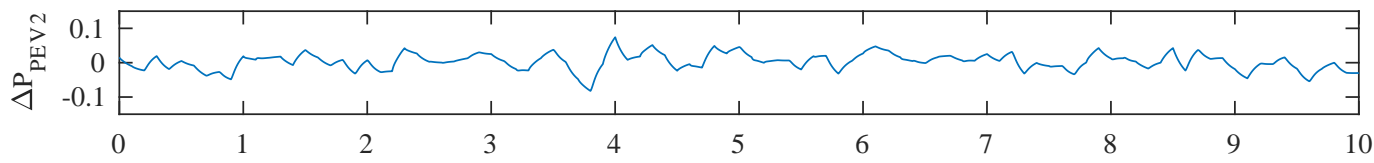

(b) Time (s)

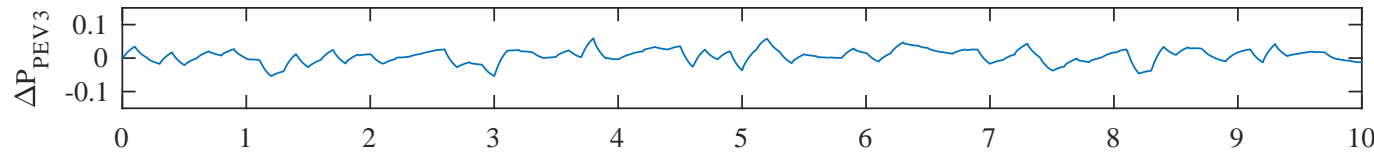

(c) Time (s)

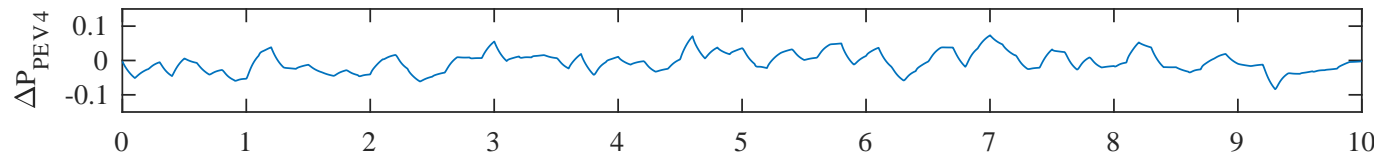

(d) Time (s)

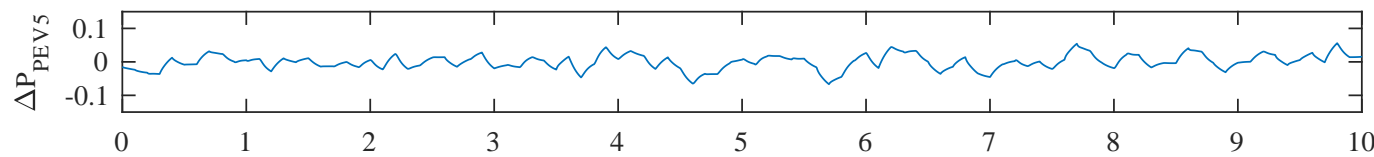

(e) Time (s)

Figure 17. Transient power output of PEV with stochastic disturbance. (a) Dynamic response of $P E V_{1}$; (b) dynamic response of $P E V_{2}$; (c) dynamic response of $P E V_{3} ;$ (d) dynamic response of $P E V_{4}$;

(e) dynamic response of $P E V_{5}$.

Therefore, the simulation results declare the effectiveness of the proposed control strategy. It can be obviously found that microgrid based on DCS-ILADRC has better anti-disturbance capability. The ILADRC-based controllers directly control the PEVs to eliminate the frequency deviation, which gives full play to the PEVs' advantages and avoids the negative effects caused by time delay. With the supplementary calculation of CPR, PEVs can contribute to the frequency regulation within their normal operating condition. The aforementioned aspects further demonstrate the effectiveness of the proposed control strategy.

\section{Conclusions}

In this paper, a novel fully-distributed control strategy is proposed to achieve fast frequency regulation of an islanded microgrid and effective coordination control of distributed energy sources. The plug-in electric vehicles are regarded as mobile battery energy storages and provide a valuable contribution to frequency regulation. Different from most of the previous works, this paper proposes a new performance indicator named the controllable power rate and combines the CPR with local controllers of PEVs to protect the system from the negative effect caused by time delay. This also guarantees the effectiveness of PEVs in the frequency regulation. Furthermore, the improved linear active disturbance rejection algorithm employed in this paper provides great anti-disturbance capability, which makes it possible for PEVs with stochastic and fluctuating power output to contribute to the frequency regulation. Finally, the two-level multi-agent system consisting of a plug-in electric vehicle multi-agent system and a diesel generator multi-agent system ensures that PEVs can operate at the maximum charging rate most of the time. In contrast, many previous works only used PEVs for frequency regulation without considering people's requirements of PEVs. Sufficient simulations, which were carried out in Section 5, demonstrate the effectiveness of the proposed distributed control strategy. 
Author Contributions: Data curation, X.Q. Formal analysis, X.Q. Funding acquisition, H.L., G.Z. and Z.W. Investigation, X.Q., Y.B., H.L. and Y.Z. Methodology, X.Q. Project administration, X.Q. Resources, X.Q. Software, X.Q. Supervision, X.Q. and Y.B. Validation, X.Q. Visualization, X.Q. Writing, original draft, X.Q. Writing, review and editing, X.Q.

Funding: This research was funded by the National Key R \& D Program of China, Grant Number NO.2017YFB0902100.

Conflicts of Interest: The authors declare no conflict of interest.

\section{References}

1. Kaur, A.; Kaushal, J.; Basak, P. A review on microgrid central controller. Renew. Sust. Energ. Rev. 2016, 55, 338-345. [CrossRef]

2. Pandey, S.K.; Mohanty, S.R.; Kishor, N. A literature survey on load-frequency control for conventional and distribution generation power systems. Renew. Sust. Energ. Rev. 2013, 25, 318-334. [CrossRef]

3. Das, D.C.; Roy, A.K.; Sinha, N. GA based frequency controller for solar thermal-diesel-wind hybrid energy generation/energy storage system. Int. J. Electr. Power Energy Syst. 2012, 43, 262-279. [CrossRef]

4. Liu, S.C.; Wang, X.Y.; Liu, P.X. Impact of Communication Delays on Secondary Frequency Control in an Islanded Microgrid. IEEE Trans. Ind. Electron. 2015, 62, 2021-2031. [CrossRef]

5. Coelho, E.A.A.; Wu, D.; Guerrero, J.M.; Vasquez, J.C.; Dragicevic, T.; Stefanovic, C.; Popovski, P. Small-Signal Analysis of the Microgrid Secondary Control Considering a Communication Time Delay. IEEE Trans. Ind. Electron. 2016, 63, 6257-6269. [CrossRef]

6. Coelho, V.N.; Cohen, M.W.; Coelho, I.M.; Liu, N.; Guimaraes, F.G. Multi-agent systems applied for energy systems integration: State-of-the-art applications and trends in microgrids. Appl. Energy 2017, 187, 820-832. [CrossRef]

7. Liu, W.; Gu, W.; Sheng, W.X.; Meng, X.L.; Wu, Z.J.; Chen, W. Decentralized Multi-Agent System-Based Cooperative Frequency Control for Autonomous Microgrids With Communication Constraints. IEEE Trans. Sustain. Energy 2014, 5, 446-456. [CrossRef]

8. Xu, Y.; Li, Z. Distributed Optimal Resource Management Based on the Consensus Algorithm in a Microgrid. IEEE Trans. Ind. Electron. 2015, 62, 2584-2592. [CrossRef]

9. Wang, Z.; Wu, W.; Zhang, B. A Fully Distributed Power Dispatch Method for Fast Frequency Recovery and Minimal Generation Cost in Autonomous Microgrids. IEEE Trans. Smart Grid 2016, 7, 19-31. [CrossRef]

10. Olfati-Saber, R.; Fax, J.A.; Murray, R.M. Consensus and Cooperation in Networked Multi-Agent Systems. Proc. IEEE 2007, 95, 215-233. [CrossRef]

11. Xia, S.W.; Bu, S.Q.; Luo, X.; Chan, K.W.; Lu, X. An Autonomous Real-Time Charging Strategy for Plug-In Electric Vehicles to Regulate Frequency of Distribution System With Fluctuating Wind Generation. IEEE Trans. Sustain. Energ. 2018, 9, 511-524. [CrossRef]

12. Rahman, M.S.; Oo, A.M.T. Distributed multi-agent based coordinated power management and control strategy for microgrids with distributed energy resources. Energy Convers. Manag. 2017, 139, 20-32. [CrossRef]

13. Zhao, T.Q.; Ding, Z.T. Distributed Initialization-Free Cost-Optimal Charging Control of Plug-In Electric Vehicles for Demand Management. IEEE Trans. Ind. Inform. 2017, 13, 2791-2801. [CrossRef]

14. Qi, X.; Bai, Y. Improved Linear Active Disturbance Rejection Control for Microgrid Frequency Regulation. Energies 2017, 10, 1047. [CrossRef]

15. Aliabadi, S.F.; Taher, S.A.; Shahidehpour, M. Smart Deregulated Grid Frequency Control in Presence of Renewable Energy Resources by EVs Charging Control. IEEE Trans. Smart Grid 2018, 9, 1073-1085. [CrossRef]

16. Senjyu, T.; Nakaji, T.; Uezato, K.; Funabashi, T. A hybrid power system using alternative energy facilities in isolated island. IEEE Trans. Energy Convers. 2005, 20, 406-414. [CrossRef]

17. Lee, D.J.; Wang, L. Small-Signal Stability Analysis of an Autonomous Hybrid Renewable Energy Power Generation/Energy Storage System Part I: Time-Domain Simulations. IEEE Trans. Energy Convers. 2008, 23, 311-320. [CrossRef]

18. Dreidy, M.; Mokhlis, H.; Mekhilef, S. Inertia response and frequency control techniques for renewable energy sources: A review. Renew. Sust. Energy Rev. 2017, 69, 144-155. [CrossRef]

19. Mauricio, J.M.; Marano, A.; Gomez-Exposito, A.; Ramos, J.L.M. Frequency Regulation Contribution Through Variable-Speed Wind Energy Conversion Systems. IEEE Trans. Power Syst. 2009, 24, 173-180. [CrossRef] 
20. Yang, J.; He, L.F.; Fu, S.Y. An improved PSO-based charging strategy of electric vehicles in electrical distribution grid. Appl. Energy 2014, 128, 82-92. [CrossRef]

21. Singh, M.; Kumar, P.; Kar, I. Implementation of Vehicle to Grid Infrastructure Using Fuzzy Logic Controller. IEEE Trans. Smart Grid 2012, 3, 565-577. [CrossRef]

(C) 2018 by the authors. Licensee MDPI, Basel, Switzerland. This article is an open access article distributed under the terms and conditions of the Creative Commons Attribution (CC BY) license (http:/ / creativecommons.org/licenses/by/4.0/). 